\title{
HHT Based Analysis on Experiments of Rotor System with Rub-Impact
}

\author{
Yong Yang ${ }^{1}$, Shuai Zhang ${ }^{2}$, Yan-huai Qu ${ }^{2}$ \\ 1. School of Information and Electric Engineering, Shenyang Agricultural University, \\ Shenyang, 110866, China \\ 2.School of Information and Electric Engineering, Shandong Institute of Business and \\ Technology, Yantai, 264005, China
}

\begin{abstract}
Keywords: rotor system, rub-impact at fixed limiter, Hilbert-Huang transform, time-frequency property
\end{abstract}

\begin{abstract}
Based on the demand of engineering, the motion characteristics and time-frequency properties of rotor system with rub-impact at fixed limiter are studied by experiment. Three typical cases at different rotating speeds are chosen from the experiment. On the basis of traditional amplitude spectrum and shaft center orbit methods, Hilbert-Huang transformation is applied to the fault signals analysis. Fault features are characterized completely and accurately by Hilbert spectrum. It is show that the vibration of the system is with typical characteristic of periodic motions and corresponding time-frequency (instantaneous frequency) -amplitude /energy representations in the rotor system with rub-impact at fixed limiter.
\end{abstract}

\section{Introduction}

Rotor-to-stator rub-impact is a type of serious fault occurring often in rotating machinery. In many cases, the rubbing fault only happens in particular position of rotating machinery [1], while the rubbing position is always fixed in most engineering and model experiment. Therefore, it is of practical significance to experiment research on rotor system with rub-impact at fixed limiter.

In many situations, the rubbing fault is distinguished depending on spectrums or the axis orbit shape. However, as a case of vibration-impact, a rotor system with rub-impact behaves in a complex nonlinearity due to some factors change caused by impact and friction during the rubbing, such as damping, stiffness, elastic force and so on. And corresponding vibration signals show time-varying and non-stationary characteristics, which include complicated fault information. While depending on the traditional spectrum and shaft centerline orbit analysis, only some rough results are obtained, and some significant characteristics including instantaneous variation used to present fault frequency/instantaneous frequency, amplitude, time and joint distribution could not be described.

In recent years, the advantages of non-stationary signals analysis method based on signal processing are presented in fault signal analysis field, of which the wavelet transform and Hilbert-Huang transform may be the best available ones [2-10]. However, wavelet transform still have its own some inevitable deficiencies and limitations [5], for instance, constant multiresolution and without self-adaptability. And HHT is proposed as an offset to these deficiencies, which is a novel adaptive signal analysis approach and can be decomposed adaptively by the signal itself which make instantaneous frequency own physical significance and gain an energy-frequency-time distribution used to reflect system inherent properties. Compared to the Fourier-based linear and stationary spectral analysis and other time-frequency analysis methods, the Hilbert-Huang transform was considered a great breakthrough and widely used in Fault Detection and Diagnosis (FDD) filed [6-8].

Rub-Impact fault at Fixed Limiter in rotor system is investigated by experiment in this paper, combined with engineering practice. Three typical cases at different rotating speeds, including $57.5 \mathrm{~Hz}, 72 \mathrm{~Hz}, 80 \mathrm{~Hz}$ are chosen from the experiment. And then HHT is applied to decompose 
and extract the fault signals. Furthermore corresponding time-frequency (instantaneous frequency) -amplitude /energy representations are obtained by Hilbert spectrum, which can present completely and accurately fault features and provide reference to further research in engineering.

\section{Hilbert-Huang Transform}

Hilbert-Huang transform (HHT), has been proposed by Huang et al [11], which consists of two parts: empirical mode decomposition (EMD) and Hilbert spectral analysis (HSA).

EMD method: the sifting process. During the HHT method, the Intrinsic Mode Function (IMF) is defined to compute instantaneous frequency more accurately. The IMFs, which are regarded as both the amplitude modulation and the frequency modulation, satisfy the following requirements: (1) the number of extremes and the number of zero crossings in the IMF must either be equal or different at most by one; and (2) at any point the mean value of the envelopes defined by the local maxima and local minima must be zero.

Based on the EMD, any complicated signals can be decomposed into a finite and often small number of 'Intrinsic Mode Functions' (IMFs) that admit well-behaved Hilbert transforms. The decomposition is adaptive, and, therefore, highly efficient. And with the Hilbert transform, the IMFs yield instantaneous frequencies as functions of time that give sharp identifications of embedded structures. The final presentation of the results is an energy-frequency-time distribution, designated as the Hilbert spectrum, and furthermore obtaining the marginal spectrum. Compared to the Fourier-based linear and stationary spectral analysis and other time-frequency analysis methods, the Hilbert-Huang transform was considered a great breakthrough and widely used in FDD filed.

Given an original signal $x(t)$, the process to EMD can be summarized as the following steps:

(1) Identification of all local extrema (maxima and minima) in the input signal $x(t)$.

(2) Creation of the upper and lower envelop by cubic spline interpolation among all the local maxima and minima, respectively.

(3) Calculate the mean of the upper and lower envelopes, which is defined as $m(t)$.

(4) Subtraction of $m_{1}(t)$ from the original input signal to obtain an IMF candidate $c(t)=x(t)-m(t)$

(5) Check the properties of $c(t)$ : If the sifting result $c(t)$ is an IMF, evaluate the residue $r(t)=x(t)-c(t)$, otherwise, replace $x(t)$ with $c(t)$ and repeat the procedure from step (1).

(6) Repeat the procedure from steps (1) to (5) by sifting the residual signal. The sifting process ends when the residue $r(t)$ satisfies a predefined stopping criterion.

At the end of the procedure we obtain a residue $r(t)$ and a collection of $\mathrm{n}$ IMFs, named $c_{i}(t)$ $(i=1,2, \ldots n)$. Furthermore the input signal $x(t)$ can be exactly reconstructed by a linear superposition:

$$
x(t)=\sum_{i=1}^{n} c_{i}(t)+r_{n}(t)
$$

Hilbert spectral analysis: Hilbert spectrum and the marginal spectrum. Having obtained the IMFs using EMD method, one applies the Hilbert transform to each IMF component,

$$
H\left[c_{i}(t)\right]=\frac{1}{\pi} \int_{-\infty}^{\infty} \frac{c_{i}(\tau)}{t-\tau} \mathrm{d} \tau
$$


After the Hilbert transform $c_{i}(t)$ and $H\left[c_{i}(t)\right]$ together form a complex signal, and compute the instantaneous frequency and amplitude according to

$$
a_{i}(t)=\sqrt{c_{i}^{2}(t)+\left(H\left[c_{i}(t)\right]\right)^{2}}, \quad \Phi_{i}(t)=\arctan \frac{H\left[c_{i}(t)\right]}{c_{i}(t)}, \quad \omega_{i}(t)=\frac{\mathrm{d} \Phi_{i}(t)}{\mathrm{d} t}
$$

Finally, the original signal $x(t)$ can be written as

$$
x(t)=\operatorname{Re} \sum_{i=1}^{n} a_{i}(t) \exp \left(i \int \omega_{i}(t) d t\right)
$$

where the residue $r_{n}(t)$ has been left out, Re $\{$.$\} denotes the real part of a complex quantity.$

Thus, the instantaneous amplitude and frequency can be expressed as functions of time in a three-dimensional plot. The frequency-time distribution of amplitude is designated as the Hilbert spectrum, $H(\omega, t)$.

\section{Experimental analysis of Rotor System with Rub-Impact at Fixed Limiter}

Experiment of Rotor System with Rub-Impact at Fixed Limiter. A test rig and mechanical model of rotor system with rub-impact at fixed limiter is depicted in Fig. 1.

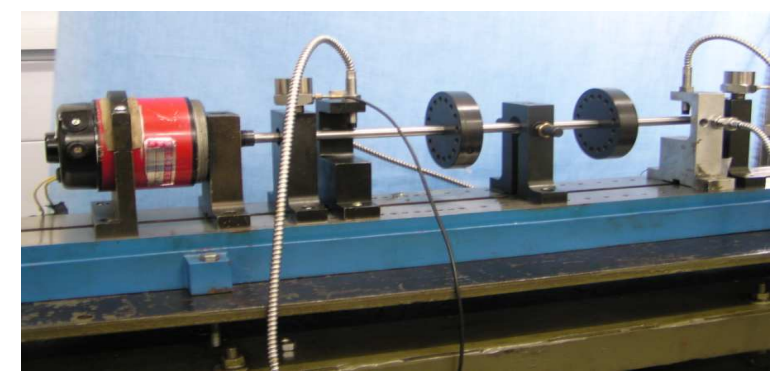

Fig. 1. The test rig and mechanical model of rotor system with rub-impact occurring on a limiter

The test rig is composed of a shaft with a total length 430 and two rigid disks, A and B, whose diameter and width are 100 and $20 \mathrm{~mm}$. The mass of two disks are $0.91364 \mathrm{~kg}$ and $0.9383 \mathrm{~kg}$, respectively. A bolt with the mass of $1 \mathrm{~g}, m_{2}=1 \mathrm{~g}$, is installed on the top of the B disk. The eccentric moment is calculated to be $m_{2} r=0.4 \times 10^{-4} \mathrm{Kgm}$, where the eccentric radius is $\mathrm{r}=40 \mathrm{~mm}$. In experiment, we alter the distance between rod which is a copper rubbing rod installed in the axial location of $20 \mathrm{~mm}$ from the B disk and shaft to simulate rub-impact fault, and the rub-impact clearance is $\delta=3.8 \times 10^{-5} \mathrm{~m}$.

The first and second natural frequencies of the rotor system are calculated and found to be $34.13 \mathrm{~Hz}$ and $124.82 \mathrm{~Hz}$ respectively. The operating speeds of the test rig are all within the range from the first critical speed to the second, and so the rotor system can be considered as a flexible system with two rigid supports. We tested both the horizontal and vertical signals under the three typical cases at different rotating speeds, including $57.5 \mathrm{~Hz}, 72 \mathrm{~Hz}, 80 \mathrm{~Hz}$ are chosen in experiment. The sampling frequency is $1000 \mathrm{~Hz}$, sampling time is $4 \mathrm{~s}$ and the sampling numbers is 4000 .

Rubbing always happened in horizontal without any impacts to the signals in vertical, so only the signals in horizontal are analyzed in this paper. In addition, corresponding the time domain waveforms and the results of EMD only in the part of the time ranging from $0.6 \mathrm{~s}$ to $1 \mathrm{~s}$ are presented in this paper for the analysis results more clearly.

Corresponding time domain waveforms, amplitude spectra in the three cases are shown in Fig.2. From Fig.2 (a)-(c), we can see that when the rotating speeds is at $57.5 \mathrm{~Hz}$ or $72 \mathrm{~Hz}$, the fault characteristics are lightly and hard to be distinguished. While only at the rotating speed $80 \mathrm{~Hz}$, some 
changes in waveforms caused by the fault can be seen. According to the amplitude spectrums and shaft center orbits, we can judge roughly: (1) when the rotating speed is at $57.5 \mathrm{~Hz}$, the amplitude spectrum is mainly in fundamental component and the shaft center orbit is approximate ellipse. Due to the rotating speed lower relatively, the degree of friction and impact between the shaft and the rubbing rod is lightly. It is indicated that system exits unbalance mainly and presents single-cycle motion characteristics. (2) when the rotating speed is at $72 \mathrm{~Hz}$, the degree of friction and impact between the shaft and the rubbing rod is deepen and $2 \mathrm{x}$ frequency component is found in the amplitude spectrum. The shaft center orbit is approximate dual-ellipse. It is indicated that system presents the double period motion characteristics. (3) When the rotating speed gets to $80 \mathrm{~Hz}$ increased, fractional frequency components and frequency multiplication components of the different degree are shown in the amplitude spectrum. Although the shaft center orbit is still approximate dual-ellipse, more confusion. It is indicated that system presents the quasi-period motion characteristics.
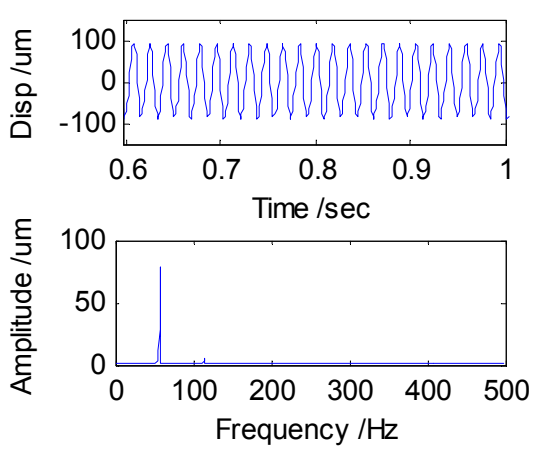

(a) $57.5 \mathrm{~Hz}$
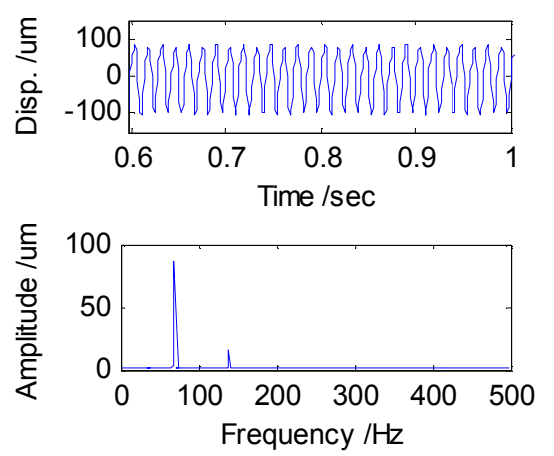

(b) $72 \mathrm{~Hz}$
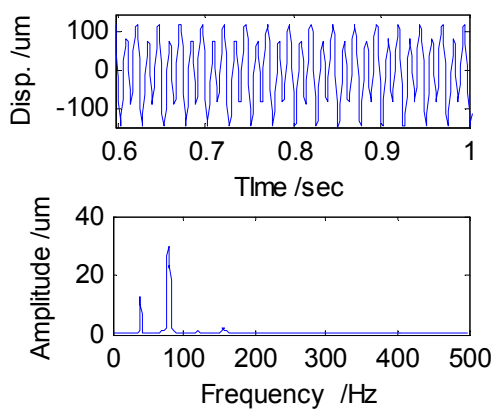

(c) $80 \mathrm{~Hz}$

Fig. 2. Time domain waveform and Amplitude spectra of the rotor system with rub-impact :

Experimental fault signals analysis based on HHT. From the result above, we can see that the time domain waveform, amplitude spectrum and shaft center orbit methods can only reflect system fault characteristics and severity roughly. Now we utilize HHT to the same three cases and obtain the results of EMD, Hilbert spectrum and the marginal spectrum shown in Fig.3-5.

We can gain the time variation characteristic existing in fault signals for revealing the fault from these results, including simple frequency component, instantaneous frequency characteristic and time-frequency (instantaneous frequency) -amplitude /energy representations. (1) When the rotating speed is at $57.5 \mathrm{~Hz}$, the results of EMD shown in the Fig.4 (a) present the fault signals are decomposed three IMF components $c 1, c 2, c 3$ and a residue $r_{n}$, in which $c 1$ is high-frequency component present mainly amplitude-modulated property, $\mathrm{c} 2$ displays periodic changes of system and c3 is low-frequency component caused by rubbing. Furthermore Hilbert real-time Spectrum shown in 5(a) give a fluctuant frequency band around the fundamental frequency, which stands for saltation and fluctuation of instantaneous frequency. And the marginal spectrum shown in Fig. 6(a) give energy representations of the every frequency point nearby the fundamental frequency, form which we can acquire accumulation and change of instantaneous frequency in the whole signals clearly cannot be characterized by the conventional spectrum methods. (2) When the rotating speed is at $72 \mathrm{~Hz}$, the results from HHT analysis are more different and complex than the case (1). Firstly, the fault signals are decomposed four IMF components, in which c1,c2 is mainly amplitude-modulated components. At the same time, two fluctuant frequency bands are shown in Hilbert real-time Spectrum (Fig.5 (b)) which presents system own double period motion characteristics, coinciding with the results from the shaft center orbit analysis. However, the Hilbert 
real-time Spectrum analysis is more accurate the shaft center orbit analysis. Moreover, we can also see two frequency bands with different amplitudes which have wider scope than case (1) in the marginal spectrum (Fig.6 (b)). It is illustrated that the degree of friction and impact between the shaft and the rubbing rod is increased to rubbing fault more heavily. (3) When the rotating speed is at $57.5 \mathrm{~Hz}$, the results of EMD consist of five IMFs. There exist obvious differences than cause (1) (2), showing frequency attenuation which illustrate the force of impact and friction growing and rubbing fault more serious. Furthermore, the corresponding marginal spectrum exhibits wider fundamental frequency bands and lager amplitude energy in fractional frequency. In addition, there still exist some frequency multiplication components. Rotor system presents the quasi-period tendency.

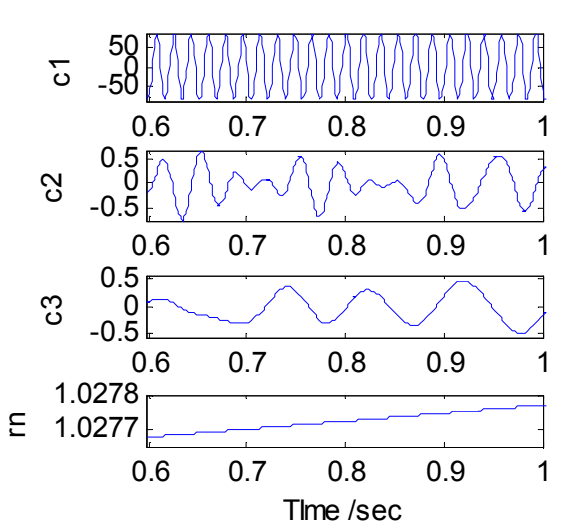

(a) $57.5 \mathrm{~Hz}$

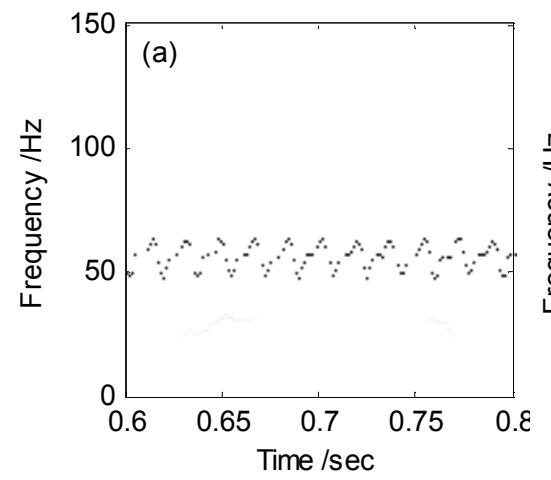

(a) $57.5 \mathrm{~Hz}$

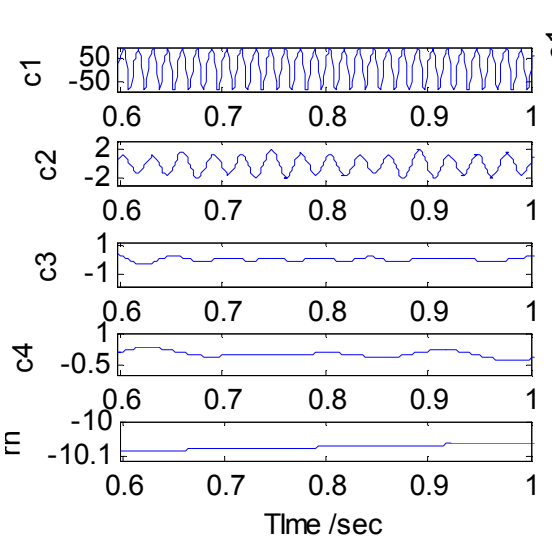

(b) $72 \mathrm{~Hz}$

Fig. 3. The results of EMD

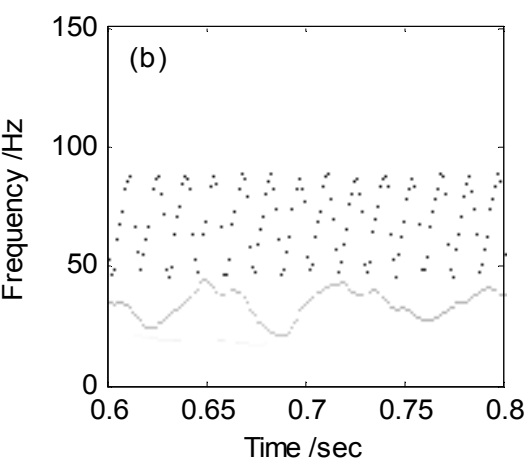

(b) $72 \mathrm{~Hz}$

Fig. 4. The Hilbert spectrum

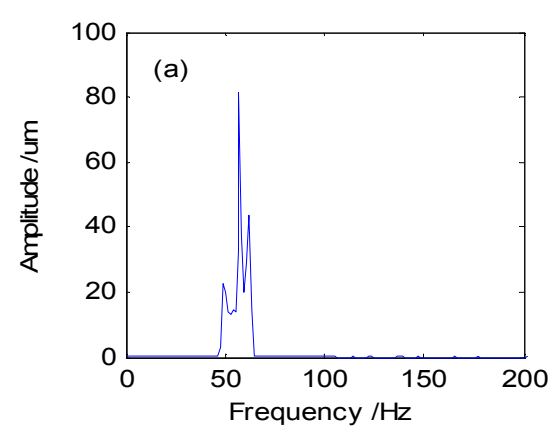

(a) $57.5 \mathrm{~Hz}$

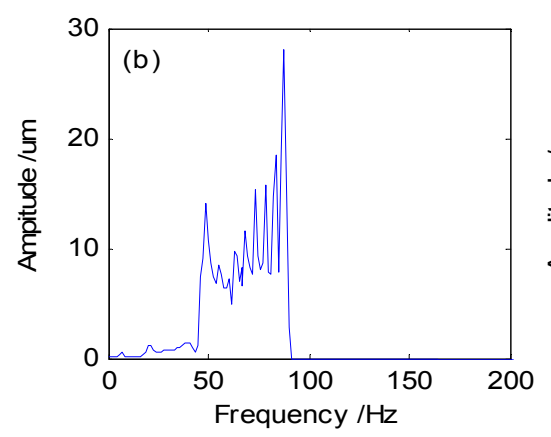

(b) $72 \mathrm{~Hz}$

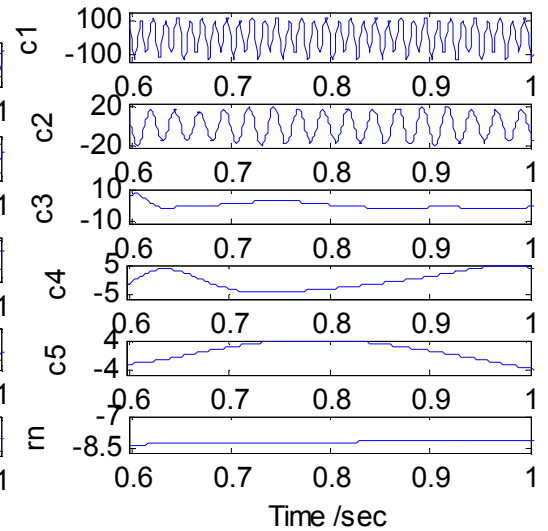

(c) $80 \mathrm{~Hz}$

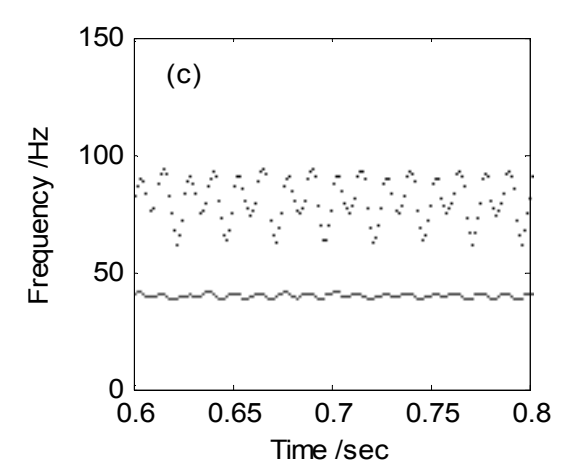

(c) $80 \mathrm{~Hz}$

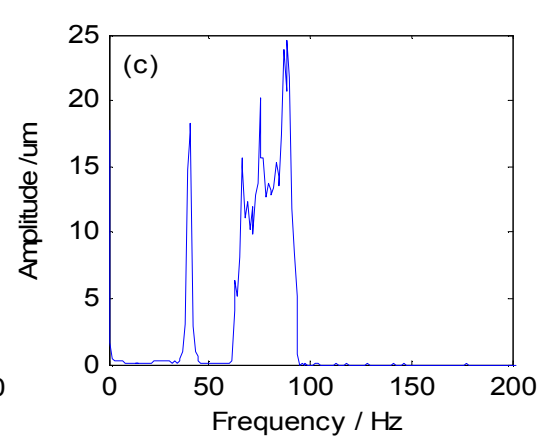

(c) $80 \mathrm{~Hz}$

Fig. 5. The Hilbert marginal spectrum 


\section{Conclusions}

In this paper, rub-impact fault at fixed limiter in rotor system is investigated by experiment. Three typical cases, at different rotating speeds $57.5 \mathrm{~Hz}, 72 \mathrm{~Hz}, 80 \mathrm{~Hz}$, are chosen and analyzed applying HHT method, which describe fault characteristics of rotor system with rubbing. Under the different rotating speeds, the degree of friction and impact between the shaft and the rubbing rod is different, and system shows various motion characteristic, instantaneous frequency change and time-frequency-amplitude representations. From the results of EMD, HAS, rotor system with rub-impact at fixed limiter have periodicity strongly, including single, double, quasi period. In addition, there are remarkable properties in frequency variation: the case which the system presents single or double period motion characteristics has corresponding periodic frequency band; while the case which the system presents quasi period motion characteristic not only has corresponding periodic frequency band, but also has low frequency band approximating a straight line, and the fluctuation of the instantaneous frequency is more remarkable. Moreover, amplitude distribution of the instantaneous frequency is corresponded to the change of frequency.

\section{References}

[1] Yu Tao Han Qing-kai Sun Wei Wen Bang-chun . Precise Diagnosis of Rotor Rubbing Fault Based on Modal Expansion and Harmonic Decomposition. Journal of Northeastern University: Natural science, 2006, 27(5):524-527.

[2] Peng Z, Chu F, Peter W. Tse. Detection of the rubbing caused impacts for rotor-stator fault diagnosis using reassigned scalogram [J]. Mechanical Systems and Signal Processing, 2005, 19: 391-409.

[3] Han Q K, Yao H. L, Zhang Z W, et al. Experiment of oil-film whirl in rotor system and wavelet fractal analyses, ASME 2005 International Design Engineering Technical Conferences, 2005, California USA.

[4] Ma H, Yu T, et al. Time-frequency features of two types of coupled rub-impact faults in rotor systems. Journal of sound and vibration, 2009, 321:109-1128.

[5] Peng Z, Chu F, He Y. Vibration signal analysis and feature extraction based on reassigned wavelet scalogram. Journal of Sound and Vibration, 2002. 253: 1087-1100.

[6] Cheng S J, Yu D J, Yang Y. The application of the energy operator demodulation approach based on EMD in machinery fault diagnosis. Mechanical Systems and Signal Processing, 2007, 21:668-677.

[7] Li Hui Zheng Haiqi Tang Liwei . Study on fault diagnosis of gear crack based on EMD and power spectrum analysis. Journal of Vibration and Shock, 2006, 25 (1): 133-136.

[8] Kang Haiying Luan Junying Zheng Haiqi Bi Xiaoyuan. Fault Diagnosis of Bearing Based on Order Tracking and HHT Marginal Spectrum. Journal of Vibration and Shock, 2007, 26(6):1-3.

[9] Peng Z K, Peter W. Tse, Chu F L. A comparison study of improved Hilbert-Huang transform and wavelet transform: Application to fault diagnosis for rolling bearing. Mechanical Systems and Signal Processing, 2005, 19:974-988.

[10] Han Q K, Zhang Z W, Wen B C. Periodic motions of a dual-disc rotor system with rub-impact at fixed limiter.Journal of Mechanical Engineering Science, 2008, 222:1935-1946.

[11]Huang N, Shen Z, Long S, et al.The empirical mode decomposition and the Hilbert spectrum for nonlinear and non-stationary time series analysis, Proceeding of the Royal Society of London Series A- Mathematical Physical and Engineering Sciences, 1998, 454: 903-995. 